\title{
The Effect of Pristine and Pegylated Graphene Oxide Nanosheets on the Functions of Human Neutrophils
}

Corresponding Author: Valeria Timganova; email:

timganovavp@gmail.com

\section{Dates}

Published 13 January 2022

Publishing services provided by Knowledge E

(c) Valeria Timganova et al. This article is distributed under the terms of the

Attribution License, which permits unrestricted use and redistribution provided that the original author and source are credited.

Selection and Peer-review under the responsibility of the 8th Scientific and Practical Conference Conference Committee.

\section{Valeria Timganova*, Svetlana Zamorina, Maria Bochkova, Anton Nechaev, Pavel Khramtsov, Kseniya Shardina, Sofia Uzhviyuk, and Mikhail Rayev}

Institute of Ecology and Genetics of Microorganisms, Ural Branch of the Russian Academy of Sciences - branch of the Perm Federal Research Center, Perm, Russian Federation ORCID

Valeria Timganova; 0000-0003-4581-1969

Abstract. Graphene oxide (GO) is very useful for biomedicine, due to its physicochemical properties; therefore, its interaction with cells of the immune system has beenextensively studied. Many studies have aimed toreduce the undesirable effects of GO through chemical modification, including through polyethylene glycol (PEG) coating. Neutrophils are the first to respond to foreign object invasion in the body. Their main functions are the uptake and destruction of foreign particles, including with the help of reactive oxygen species (ROS).Our study aimed to investigate theengulfment of unmodified graphene oxide (GO) and graphene oxide coated with polyethylene glycol (GO-PEG) by human neutrophils and the effect of nanosheets on the production of ROS.We used sheets of GO (Ossila, Great Britain, average plate size $1-5 \mu \mathrm{m})$ and GO-PEG (569 $\pm 14 \mathrm{~nm}, \mathrm{PEG}$ coating $\approx 20 \%$ ) at concentrations of $12.5 \mu \mathrm{g} / \mathrm{mL}$, $25 \mu \mathrm{g} / \mathrm{mL}$, and $50 \mu \mathrm{g} / \mathrm{mL}$. The uptake of nanosheets was assessed by flow cytometry, taking into account the level of background adhesion of nanoparticles. ROS production was evaluated by luminol-dependent chemiluminescence (LCL).It was found that GO $(12.5 \mu \mathrm{g} / \mathrm{mL}, 25 \mu \mathrm{g} / \mathrm{mL}$, and $50 \mu \mathrm{g} / \mathrm{mL})$ was actively internalized by neutrophils, while the uptake of GO-PEG was not detected. GO and GO-PEG particles $(25 \mathrm{\mu g} / \mathrm{mLand} 50$ $\mu \mathrm{g} / \mathrm{mL}$ ) reduced the total production of ROS by human leukocytes. Thus, the modifying of GOnanosheets with PEG resulted in the abolishment of their active uptake by neutrophils but did not affect the GO inhibitory effect on their oxidative activity.

Keywords: graphene oxide surface modification, pegylated graphene oxide nanosheets, nanoparticle uptake, human neutrophils, of reactive oxygen species

\section{Introduction}

Graphene is a two-dimensional atomic-scale material, that is made of a single layer of carbon atoms, lightweight and durable. Due to its unique properties, it began to be used in many branches of science, including biomedicine [1, 2]. In biological and medical research, oxidized forms of graphene are mainly used, namely, graphene oxide (GO), since its surface is easier to modify $[3,4]$. In particular, modification of GO nanosheets with polymers helps to reduce toxicity and improve biocompatibility with cells of the 
immune system [5]. The most popular coating for $\mathrm{GO}$ is polyethylene glycol (PEG) [6]; we used it to modify nanoparticles in this work.

During the inflammatory reaction, neutrophils are the first to migrate from the bloodstream to the inflammation site, carrying out the clearance of pathogens and cellular debris, while producing many substances with antimicrobial activity, including reactive oxygen species (ROS). Because neutrophils are the most abundant leukocytes in the bloodstream, it is essential to investigate the effects of $\mathrm{GO}$ on these cells. It is known that ROS produced by neutrophils can contribute to the destruction of $G O$ nanoparticles that have entered the body [7]. At the same time, it was shown that unmodified GO is capable of exerting a cytotoxic effect, initiating an oxygen explosion in human neutrophils, which ultimately leads to apoptosis of neutrophils [8]. Neutrophils are phagocytes, and the primary type of their interaction with nanoparticles is engulfment (uptake), but the mechanisms of this process are under study. According to the available data, the uptake of graphene oxide particles proceeds rapidly, and the rate of this process depends on the type of cells, the size of the particles, and the type of their functionalization. $[9,10]$

\subsection{Objective}

Thus, this work aimed to study the engulfment of GO and GO-PEG nanosheets by neutrophils of human peripheral blood in a short time, along with their effects on ROS production.

\section{Materialsand Methods}

The study was carried out in accordance with the 2000 Helsinki Declaration of the WMA, and the Protocol of the Council of Europe Convention on Human Rights and Biomedicine 1999, permission of the Ethics Committee of the IEGM UB RAS (IRB00010009) dated 30.08.2019.

\subsection{Research Objects}

We used graphene oxide (GO) particles, size 1-5 $\mu \mathrm{m}$ (Ossila Ltd, Great Britain). The GO was modified with linear PEG, according to [11]. The average size of the GO-PEG sheetsis $569 \pm 14 \mathrm{~nm}$. The amount of PEG, covering the GO-PEG particles $\approx 19-20 \%$. The technique for the modification of GOsheets was published earlier [12]. 
We used heparinized blood of conventionally healthy donors ( $n=7$, age $35 \pm 7$ years). Leukocytes were isolated under sterile conditions by spontaneous sedimentation for 40 minutes at $\mathrm{t} 37^{\circ} \mathrm{C}$.

\subsection{Assessment of Cell Viability}

Cell viability was determined using $0.4 \%$ trypan blue (Sigma Aldrich, USA). The cells were mixed with a dye in a 1:1 ratio; after 1 minute, the percentage of dead (stained) cells was determined in a Neubauer chamber.

\subsection{Sample Preparation and Assessment of Uptake of Nanosheets by Neutrophils}

To the nanosheetuptake assessment, 0.2 million leukocytes were mixed in RPMI-1640 medium with the addition of $20 \%$ autologous serum with GO and GO-PEG in polypropylene tubes until the final concentrations of nanoparticles were reached $12.5,25$, and 50 $\mu \mathrm{g} / \mathrm{mL}$. The samples were incubated at $4^{\circ} \mathrm{C}$ and $37^{\circ} \mathrm{C}$ [13]for 30 minutes. After washing the cells with cold phosphate-buffered saline without magnesium and calcium (DPBS "Gibco", USA), they were resuspended in a small volume of DPBS with 0.5\% BSA and analyzed on a CytoFLEX S flow cytometer, gating neutrophils with high granularity and increased size by light scatter plot (SSC/FSC) and taking into account their percentage of neutrophils-singlets (Figure 1). An increase in the proportion of neutrophils with high granularity and increased size we interpreted as particle uptake. Samples without the addition of nanoparticles served as control. For further analysis, the KALUZA Analysis 2.1.1 software (Beckman Coulter, USA) was used.

\subsection{Sample Preparation and Luminol-Dependent Chemilumines- cence of Leukocytes}

Leukocytes were washed from serum with cold Hanks solution (Biolot, Russia). Then, into the wells of a 96-well sterile plate for a luminometer (Nunc, Denmark) were sequentially added: Hanks solution without phenol red (Biolot, Russia), GO or GO-PEG at concentrations of $50 \mu \mathrm{g} / \mathrm{mL} ; 25 \mu \mathrm{g} / \mathrm{mL} ; 12.5 \mu \mathrm{g} / \mathrm{mL}$, or $0 \mu \mathrm{g} / \mathrm{mL}$ (control); $10 \mu \mathrm{L}$ of cell suspension $\left(10^{6} / \mathrm{mL}\right)$, and the sodium salt of luminol (Sigma-Aldrich, United States) to a final concentration of $20 \mu \mathrm{M}$. To the stimulated LCL evaluation, opsonized zymosan $(\mathrm{OZ})$ was added to half of the wells to a final concentration of $1.5 \mathrm{mg} / \mathrm{mL}$. 

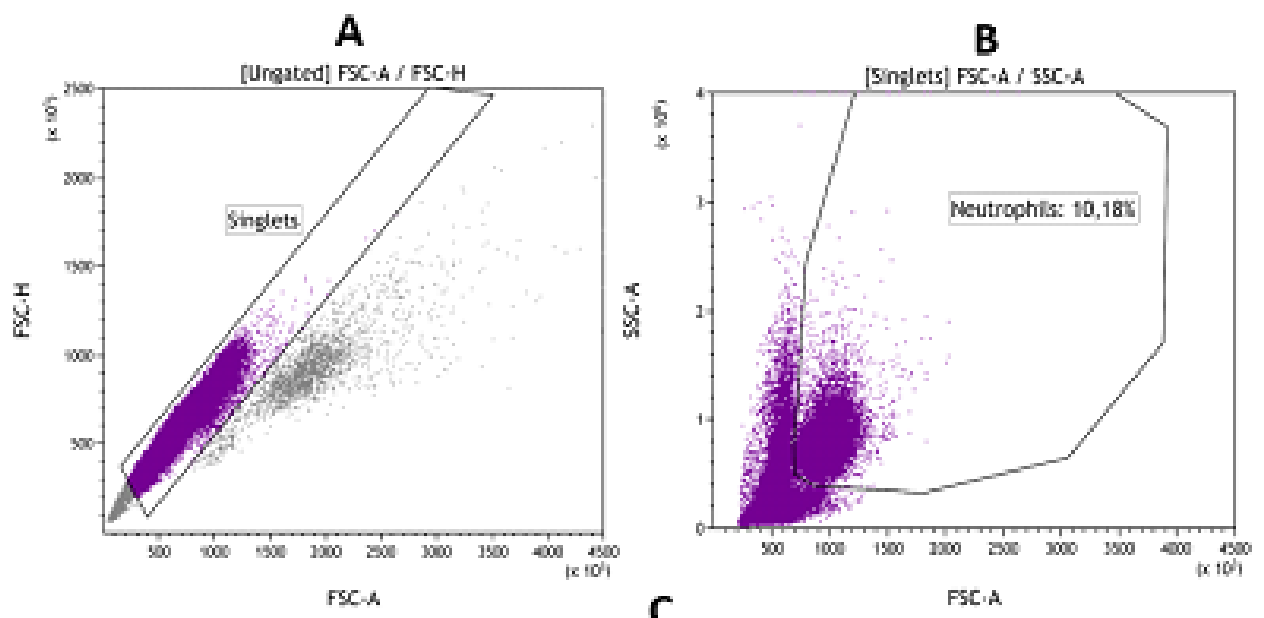

C
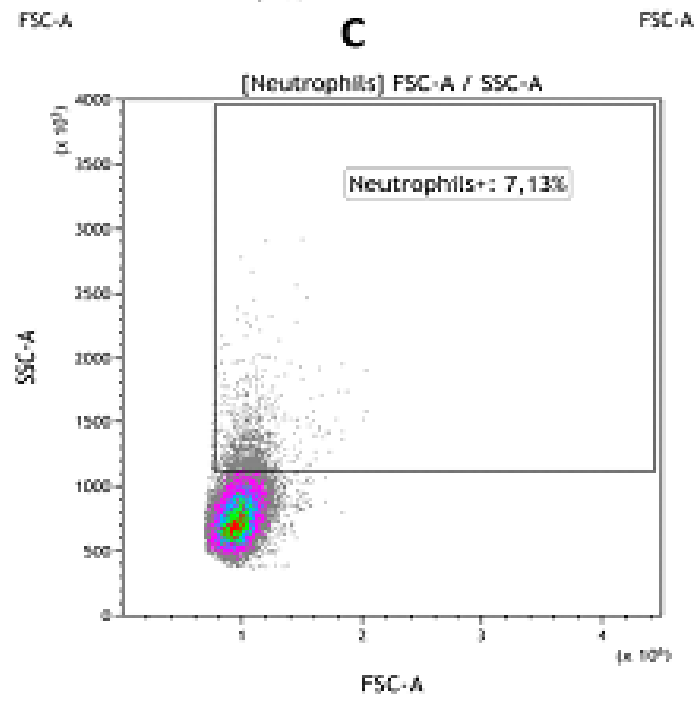

Figure 1: Example of sequential gating of large neutrophils with high granularity.

Note: $A$ - gating of singlets, $B$ - gating of neutrophils, $C$ - gating of the area of large, highly granular (engulfing particles) neutrophils.

The degree of quenching of luminescence by GO and GO-PEG particles was assessed in a cell-free system. Instead of zymosan and cells, $\mathrm{H}_{2} \mathrm{O}_{2}$ was added to the samples at concentrations of $10 \% ; 5 \% ; 2.5 \% ; 1.25 \%$; $0.625 \%, 0.312 \% ; 0.156 \% ; 0.078 \%$ and $0.039 \%$.

The luminescence intensity was measured for 30 minutes, with an interval of three minutes on a Synergy $\mathrm{H} 1$ hybrid reader (BioTek). The integral index was calculated: light sum ( $\mathrm{S}$, the area under the chemiluminescence curve).

Statistical data analysis was performed in GraphPad Prism 8, using one-way (Friedman) and two-way (Sidak's and Tuke's multiple comparisons tests) ANOVA. 


\section{Results}

\subsection{Study of the Effect of GOand GO-PEG Nanosheets on Leuko- cyte Viability}

It was shown that the percentage of living cells in short-term (30 min) cultures of human peripheral blood neutrophils was $98.5 \pm 3.4$. GO and GO-PEG nanosheets at concentrations of $12.5,25$, and $50 \mu \mathrm{g} / \mathrm{mL}$ did not have a significant effect on cell viability.

\subsection{Uptake of GOand GO-PEG Nanosheets by Human Peripheral Blood Neutrophils}

The uptake of GO and GO-PEG particles was detected by flow cytometry. The percentage of single high-granular large neutrophils was taken into account on the light scatter plot (FSC/SSC) (Fig. 1).

Engulfment is an active metabolic process that stops at low temperatures, while nonspecific binding of nanoparticles or adhesion to cell membranes under these conditions is preserved since they are not energy-dependent. However, at $37^{\circ} \mathrm{C}$, the particles can be both adhered to the cell surface and engulfed by them. To be able to separate the adhesion from the engulfment itself, half of the samples were incubated at $4 \mathrm{C}$ [13].

According to the data obtained, at $4^{\circ} \mathrm{C}$ the percentage of large, highly granular neutrophils did not change. Therefore, the adhesion of GO and GO-PEG particles on the neutrophil surface did not occur (Fig. 2).

In the samples with GO particles, a significant increase in the percentage of large, highly granular (uptaking particles) neutrophils was observed in relation to the control without particles during incubation at 37C. Besides, the percentage of large, highly granular neutrophils in samples with 12.5 and $50 \mu \mathrm{g} / \mathrm{mL}$ GO was significantly higher than that in samples incubated at $4^{\circ} \mathrm{C}$ (Fig. 2). It confirms the fact that it is the engulfment that occurs, and not only the adhesion of particles to the cell surface.

In the samples with pegylated GO particles, no significant changes were detected either in comparison with the control without particles or in relationto the samples incubated at 4C (Fig. 2).

Thus, GO particles are able to be actively uptaken by neutrophils, while pegylation prevents their internalization by cells. 
GO

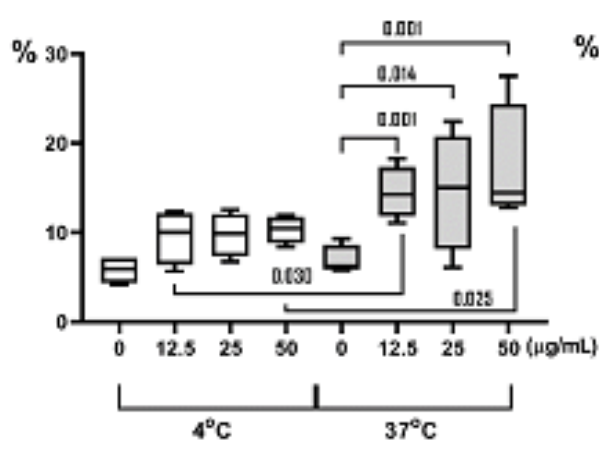

GO-PEG

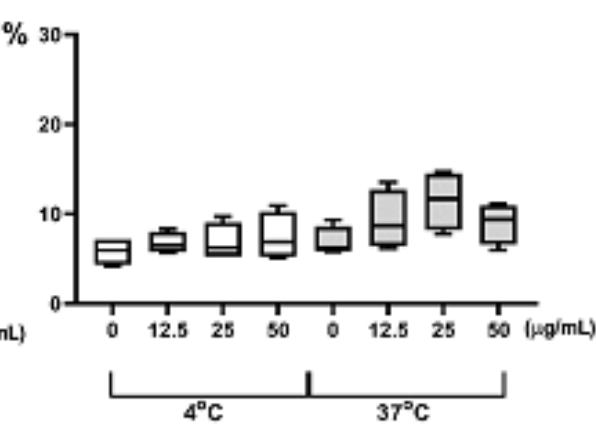

Figure 2: Adhesion $\left(4^{\circ} \mathrm{C}\right)$ and uptake $\left(37^{\circ} \mathrm{C}\right)$ of $\mathrm{GO}$ and GO-PEG nanosheets by neutrophils of human peripheral blood $(n=7)$.

\subsection{Effect of GO and GO-PEG on the Production of Reactive Oxy- gen Species by Neutrophils Detected by Luminol-Dependent Chemiluminescence}

When assessing the effect of GO and GO-PEG on spontaneous and OZ-stimulated ROS production by the LCL method, it was found that GO and GO-PEG particles at concentrations of 25 and $50 \mu \mathrm{g} / \mathrm{mL}$ reliably reduced the $\mathrm{CL}$ light sum indices relative to the controls without particles (Fig. 3 ).

In order to exclude the effect of quenching of luminescence by nanoparticles, we carried out experiments on simulating luminescence in a cell-free system (luminol + hydrogen peroxide in different concentrations). No luminescence quenching effect was detected (data not shown).

Thus, it was shown that GO and GO-PEG particles (25 and $50 \mu \mathrm{g} / \mathrm{mL}$ ) reduced the total ROS production by human leukocytes in both spontaneous and zymosan-induced test variants. Thus, the coating of $\mathrm{GO}$ particles with polyethylene glycol does not affect the processes of ROS synthesis by cells. However, the procedure for modifying the graphene oxide with polyethylene glycol leads to the cancellation of their active uptake by neutrophils.

\section{Discussion}

It is important to note that when any nanoparticles come into contact with neutrophils, we, first of all, face the internalization (uptake) of nanoparticles, since these cells are capable of phagocytosis. Rapid clearance of particles from the bloodstream is 
A
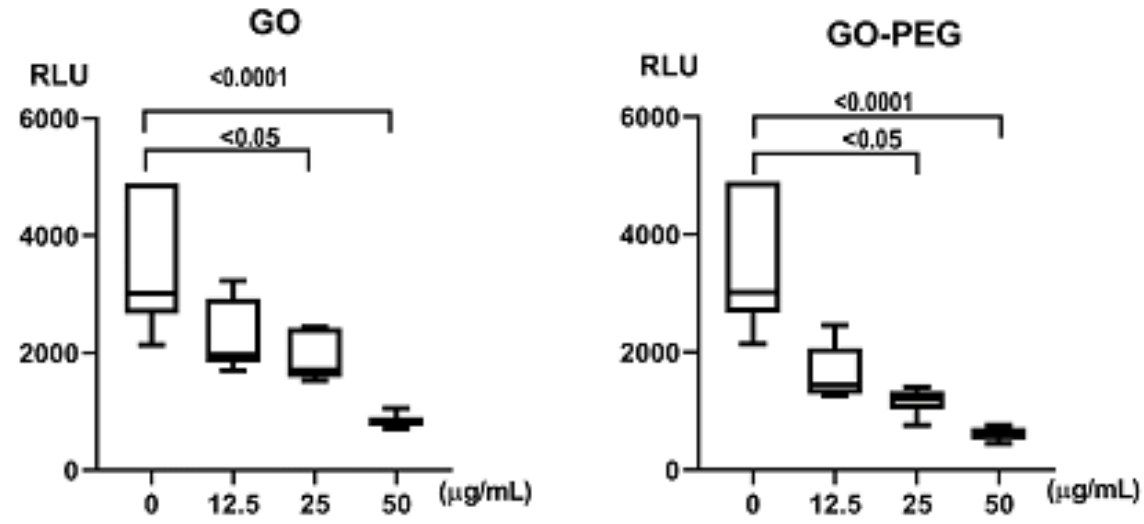

B

GO+zymosan

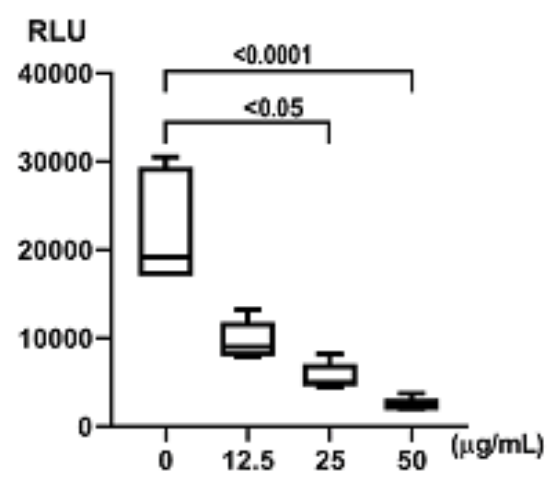

GO-PEG+zymosan

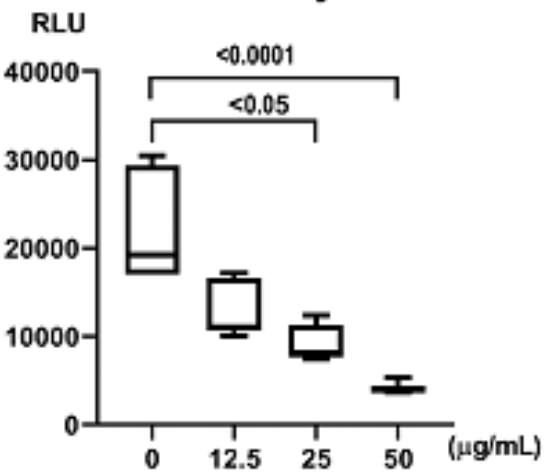

Figure 3: Effect of GO and GO-PEG on the indices of the light sum of chemiluminescence in spontaneous (A) and stimulated (B) LCL ( $\mathrm{n}=7$ ).

undesirable when it comes to, for example, targeted delivery of pharmaceutical or biological preparations. The coating of nanoparticles and, in particular, nanosheets of GO with polymers such as PEG prolongs their residence time in the body. It may be favorable for the development of innovative, effective biopharmaceuticals. However, from the point of view of fundamental immunology, this area needs additional research. Despite the abundance of data concerning the uptake of graphene nanoparticles, carbon nanotubes, and fullerenes by cells, the mechanism of their internalization and the consequences of their use for the body remain poorly understood. In addition to the internalization of nanoparticles, there is also a specific interaction of flat sheets of GO with cells, in which they are located in parallel manner on the membrane. This is the so-called "mask effect", which can either promote the uptake of nanoparticles or mechanically separate the cell from the environment [14]. In addition, there is evidence 
that GO cannot be entirely phagocytosed by human cells, and this phenomenon was called "frustrated phagocytosis" [8].

According to our study, 1-5 $\mu \mathrm{mGOnanoplates} \mathrm{are} \mathrm{uptaken} \mathrm{by} \mathrm{human} \mathrm{neutrophils,} \mathrm{and}$ the functionalization procedureof GO nanosheets with PEG leads to the cancellation of their active internalization. Probably, this is becausepegylationinterferes with the opsonization of particles and, consequently, their uptake by the cell [15]. However, the size and shape of particles are also important factors that determine the rate of their internalization [16]. In our case, the processofGO nanoparticles modifying with polyethylene glycol led to a decrease in their size. Therefore, it cannot be said unequivocally that only polyethylene glycol itself is responsible for the absence of rapid uptake of GO-PEG by neutrophils. In addition, the available data on the pegylationeffect on the uptake of graphene particles by cells are contradictory [10].

In our experiment, GO and GO-PEG identically reduced the light sum of LCL. Perhaps this is due to the manifestation of the antioxidant activity of $\mathrm{GO}$, the data on which are contradictory $[17,18,19]$. The interaction of $G O$ with ROS may be one of the possible reasons for a decrease in the intensity of luminescence, but this may be due to its direct effect on leukocytes. Previously, it was shown that $\mathrm{GO}$ could reduce the phosphorylation level of some intracellular signal transductors, in particular, protein kinase B and inhibit the macrophageinducible NO-synthase expression [20]. It means that a decrease in ROS production by rapidly blocking intracellular signals with $\mathrm{GO}$ is a likely scenario.

Thus, we have established that GO particles are internalized by neutrophils, while GO-PEG particles are not. The values of the LCL light sum decreased in the same way in samples with 25 and $50 \mu \mathrm{g} / \mathrm{mLGO}$ and GO-PEG. The mechanisms underlying these effects require further research.

\section{Acknowledgments}

This work was supported by a Russian Science Foundation grant № 19-15-00244.

\section{References}

[1] Razumov VF.Graphene is a new breakthrough in nanotechnology. Russian Nanotechnology. 2010;5:17-22.

[2] Pankratov D.V., Gonsales-Arribas E., Parunova Y.M. et al. New nanobiocomposite materials for bioelectronic devices. Acta Naturae. 2015;7:103-107. 
[3] Shareena D.T.P., Mcshan D., Dasmahapatra A., Tchounwou P. A review on graphenebased nanomaterials in biomedical applications and risks in environment and health. Nano-Micro Letters.2018; 10(53), 1-34 .

[4] Zhang H, Yan T., Xu S. et al. Graphene oxide-chitosan nanocomposites for intracellular delivery of immunostimulatory $\mathrm{CpG}$ oligodeoxynucleotides. Materials Science \& Engineering C-Materials for Biological Applications. 2017; 1(73),144-151.

[5] Bi S, Zhao T,Luo B. A graphene oxide platform for the assay of biomolecules based on chemiluminescence resonance energy transfer. Chemical Communications. 2012;48(1). 106-108.

[6] Tabish TA, Zhang S, Winyard PG. Developing the next generation of graphene-based platforms for cancer therapeutics: The potential role of reactive oxygen species. Redox Biology. 2018;15. 34-40

[7] Kurapati R, Russier J, Squillaci MA et al. Dispersibility-dependent biodegradation of graphene oxide by myeloperoxidase. Small. 2016;11(32). 3985-3994

[8] Paino IM, Santos F, Zucolotto V. Biocompatibility and toxicology effects of graphene oxide in cancer, normal, and primary immune cells. Journal of Biomedical Materials Research - Part A. 2017;105(3). 728-736

[9] Mendes RG, Mandarino A., Koch B. et al. Size and time dependent internalization of label-free nano-graphene oxide in human macrophages. Nano Research. 2017;10. 1980-1995

[10] Mukherjee SP, Bottini M, Fadeel B. Graphene and the immune system: A romance of many dimensions. Frontiers in Immunology. 2017;8. 673-674

[11] Hermanson GT. Bioconjugate chemistry. $2^{\text {nd }}$ edition. San Diego: Academic Press; 2008.

[12] Zamorina SA, Timganova V., Bochkova M., Khramtsov P. Effect of graphene oxide nanoparticles on the differentiation of myeloid suppressor cells.Byulleten' Eksperimental'noybiologiiimeditsiny. 2020;170(7):102-105.

[13] Santos JL, Montes MJ, Gutiérrez F, Ruiz C. Evaluation of phagocytic capacity with a modified flow cytometry technique. Immunology Letters. 1995;45. 1-4.

[14] Russier J, Treossi E, Scarsi A et al. Evidencing the mask effect of graphene oxide: A comparative study on primary human and murine phagocytic cells. Nanoscale. 2013;5. 11234-11247

[15] Sanchez L,Yi Y, YuY. Effect of partial PEGylation on particle uptake by macrophages. Nanoscale. 2017;91, 288-297 doi.org/10.1039/C6AN00325G 
[16] Bisso PW, Gaglione S, Guimarães PPG, Mitchell MJ, Langer R Nanomaterial interactions with human neutrophils. ACS Biomaterials Science and Engineering. 2018;4(12). 4255-4265 doi.org/410.1021/acsbiomaterials.8b01062

[17] Baali N., Khecha A., Bensouici A., Speranza G., Hamdouni N. Assessment of antioxidant activity of pure graphene oxide (GO) and $\mathrm{ZnO}$-decorated reduced graphene Oxide ( $\mathrm{rGO}$ ) using DPPH radical and $\mathrm{H} 2 \mathrm{O} 2$ scavenging assays. Journal of Carbon Research. 2019;5. 752-759 doi.org/10.3390/c5040075

[18] Markovic ZM, Jovanovic SP, Maškovic PZ et al. Graphene oxide size and structure pro-oxidant and antioxidant activity and photoinduced cytotoxicity relation on three cancer cell lines. Journal of Photochemistry and PhotobiologyB: Biology. 2019;200. 111647-111648

[19] Qiu Y, Wang Z, Owens AC et al. Antioxidant chemistry of graphene-based materials and its role in oxidation protection technology. Nanoscale. 2014;6(20). 11744-11755

[20] Han J, Kim YS, Lim MY et al. Dual roles of graphene oxide to attenuate inflammation and elicit timely polarization of macrophage phenotypes for cardiac repair. ACS Nano. 2018;12 (2) 1959-1977 